\title{
Context Awareness via a Single Device-Attached Accelerometer During Mobile Computing
}

\author{
Ji Soo Yi, Young Sang Choi, Julie A. Jacko \\ Laboratory of Human Computer Interaction and Health Care \\ Informatics, School of Industrial and Systems Engineering, \\ Georgia Institute of Technology \\ 765 Ferst Drive NW, Atlanta, GA 30332 USA \\ Tel: 14043852545 \\ \{jyi, ychoi, jacko\}@isye.gatech.edu
}

\author{
Andrew Sears \\ Interactive Systems Research Center, \\ Information Systems Department, UMBC \\ 1000 Hilltop Circle, Baltimore, MD 21250 USA \\ Tel: 14104553883 \\ asears@umbc.edu
}

\begin{abstract}
Interest in context-aware computing has expanded the use of sensing technologies. The accelerometer is one of the most widely used sensors for capturing context because it is small, inexpensive, lightweight, and self-operable. In efforts to obtain behavioral patterns, many studies have reported the use of multiple accelerometers attached to the human body. However, this is difficult to implement in real-life situations and may not fully address the context of user interaction. In contrast, the present study employed a single tri-axial accelerometer attached to a handheld computing device instead of to a user. The objective was to determine what contextual information could be obtained from this more feasible, albeit limited, source of acceleration data. Data analyses confirmed that changes in both mobility and lighting conditions induced statistically significant differences in the output of the accelerometer.
\end{abstract}

\section{Categories and Subject Descriptors}

H.5.2 [User Interfaces]: Interaction styles (e.g., commands, menus, forms, direct manipulation)

\section{General Terms}

Measurement; Experimentation; Human Factors

\section{Keywords}

Context-awareness; Accelerometer; Mobile computing; Pen-based handheld device; Sitting; Treadmill; Walking; Gait

\section{INTRODUCTION}

Context-aware computing aims to enable computing devices to use contextual information such as location, time, nearby objects, network bandwidth, orientation, light level, tilt, and vibration to provide more effective mobile computing solutions. (e.g., [6]). Mobile, context-aware computing offers human-computer interaction researchers new challenges due to the vast quantity of highly variable, contextual information present during user-device interactions. Examples are mobility (e.g., when devices are used while the user is on the move), lighting, and sound. Capturing contextual information can be realized through various sensor and recognition technologies.

The accelerometer is one of the most widely used sensors not only in context-aware computing, but also in various other domains, including but not limited to medical engineering (e.g., [11]), gait analysis (e.g., [7]), and human factors (e.g., [9]). The prevalence of the accelerometer is attributed to its numerous advantages over other sensors: It is lightweight, small, and inexpensive [10]; It consumes small amounts of energy [5]; It captures high-frequency movement information; It is self-operable, meaning that it does not require infrastructure for operation. Due to these advantages, the accelerometer is used as a primary sensor for capturing contextual information regarding movement and is often coupled with other technologies as a secondary/supplementary sensor (e.g., [1]).

However, the accelerometer also has several limitations. As most studies in Bao's [2] survey confirmed, one limitation is the need to employ multiple accelerometers - often attached to various locations on the human body, especially at the hip, for the best recognition of human movement. However, wearing multiple accelerometers is not an immediate and pragmatic solution for data collection in real-life mobile computing though some innovative solutions have been under development [8]. Another limitation is a lack of concrete guidelines for utilizing acceleration data. Because they are noisy time-series data, suitable descriptions of trending behaviors are difficult to derive.

Some studies (e.g., [5]) have shown that if the set of behavioral patterns is limited, a single accelerometer attached to a separate device can be useful to capture limited contextual information regarding task performance with the device. In fact, the key behavioral patterns of mobile device users may be conducive to measurement of this type. Thus, the present paper aims to examine how two representative contextual factors, mobility and lighting [4], affect data from a single accelerometer attached to a mobile device during device use.
Permission to make digital or hard copies of all or part of this work for personal or classroom use is granted without fee provided that copies are not made or distributed for profit or commercial advantage and that copies bear this notice and the full citation on the first page. To copy otherwise, or republish, to post on servers or to redistribute to lists, requires prior specific permission and/or a fee.

MobileHCI'05, September 19-22, 2005, Salzburg, Austria.

Copyright 2005 ACM 1-59593-089-2/05/0009...\$5.00. 
Table 1. The summary of demographic information of participants

\begin{tabular}{|c|c|c|c|c|c|c|c|}
\hline $\mathbf{N}$ & $\begin{array}{c}\text { Mean } \\
\text { Age } \\
\text { (SD) }\end{array}$ & Gender & $\begin{array}{c}\text { Native } \\
\text { language }\end{array}$ & $\begin{array}{c}\text { Dominant } \\
\text { hand }\end{array}$ & $\begin{array}{c}\text { Computer use } \\
\text { frequency }\end{array}$ & PDA owner? & $\begin{array}{c}\text { Regularly read } \\
\text { while walking? }\end{array}$ \\
\hline 119 & $\begin{array}{c}21.83 \\
(1.53)\end{array}$ & $\begin{array}{c}\text { Male: } \mathrm{n}=59, \\
\text { Female: } \mathrm{n}=60\end{array}$ & $\begin{array}{c}\text { English: } \mathrm{n}=98, \\
\text { Other: } \mathrm{n}=21\end{array}$ & $\begin{array}{c}\text { Right: } \mathrm{n}=113, \\
\text { Left: } \mathrm{n}=6\end{array}$ & $\begin{array}{c}\text { >1/day: } \mathrm{n}=111 \\
\approx 1 / \text { day: } \mathrm{n}=8 \\
<1 / \text { day: } \mathrm{n}=0\end{array}$ & $\begin{array}{c}\text { Current: } \mathrm{n}=21 \\
\text { Previous: } \mathrm{n}=17 \\
\text { Never: } \mathrm{n}=81\end{array}$ & $\begin{array}{c}\text { Yes: } \mathrm{n}=37, \\
\text { No: } \mathrm{n}=82\end{array}$ \\
\hline
\end{tabular}

\section{METHODOLOGY}

One hundred twenty-six undergraduate students were recruited from a senior-level undergraduate class in the School of Industrial and Systems Engineering at the Georgia Institute of Technology. They voluntarily participated in the study, and extra credit for the class was provided as compensation. Data from 119 out of 126 subjects were usable and thus analyzed. Demographic information is summarized in Table 1.

Each participant was randomly assigned to one of three groups (sitting at a desk, walking on the treadmill, and following an obstructed path on the floor). Equivalence between the three groups was verified with respect to age, gender, native language, dominant hand, computer usage, and ownership of personal digital assistant (PDA). Participants were asked to perform information retrieval tasks under two different lighting conditions (high light (260 lux) and low light (85 lux)), in a random order. Participants had chances to adjust their eyes in different lighting conditions because they were instructed to read an introduction screen on the PDA at the beginning of the tasks under each condition. Participants read passages of texts and answered multiple-choice questions using stylus input. During the tasks, participants positioned the PDA on a desk in the sitting condition and held it with one hand in the walking and treadmill conditions, respectively. Before performing the tasks in the walking and treadmill conditions, participants were calibrated at their typical walking speeds.

While subjects performed the tasks, a tri-axial accelerometer attached to the back of a PDA, Palm m505, collected the acceleration data at a sampling rate of $7 \mathrm{~Hz}$. The $\mathrm{x}-, \mathrm{y}-$, and $\mathrm{z}-$ axes of the accelerometer were aligned right/left, up/down, and forward/backward movements of the PDA, respectively. Device acceleration toward the right, up, and forward of the PDA generated positive values in $\mathrm{x}-, \mathrm{y}-$, and $\mathrm{z}-$ axial acceleration, respectively. Various measures were collected in addition to device acceleration, and are reported elsewhere [3, 4].

Table 2. The results of multivariate repeated measures ANOVA with regard to the mean of acceleration

\begin{tabular}{|c|c|r|r|r|r|}
\hline Effects & Factors & F & Hypothesis df & Error df & Sig. \\
\hline Between Subjects & Mobility & 24.10 & 6 & 228 & $<0.01 *$ \\
\hline \multirow{2}{*}{ Within Subjects } & Lighting & 4.17 & 3 & 114 & $<0.01 *$ \\
\cline { 2 - 6 } & Lighting $\times$ Mobility & 3.22 & 6 & 228 & $<0.01 *$ \\
\hline
\end{tabular}

An asterisk (“*”) denotes a statistically significant difference at level 0.05 .

Table 3. Pair-wise comparisons of the means of acceleration between mobility conditions and light conditions

\begin{tabular}{|c|c|c|c|c|}
\hline \multirow{2}{*}{ Measures } & \multicolumn{2}{|c|}{ Mobility } & \multicolumn{2}{|l|}{ Light } \\
\hline & Comparisons & p-value ${ }^{a}$ & Comparisons & p-value ${ }^{a}$ \\
\hline \multirow{3}{*}{ MAx } & Sitting $>$ Treadmill & $0.000^{*}$ & \multirow{3}{*}{ High light $=$ Low light } & \multirow{3}{*}{0.379} \\
\hline & Sitting $=$ Walking & 0.254 & & \\
\hline & Treadmill $<$ Walking & $0.000 *$ & & \\
\hline \multirow{3}{*}{ MAy } & Sitting $<$ Treadmill & $0.000 *$ & \multirow{3}{*}{ High light $<$ Low light } & \multirow{3}{*}{$0.001 *$} \\
\hline & Sitting $<$ Walking & $0.000 *$ & & \\
\hline & Treadmill $<$ Walking & $0.000 *$ & & \\
\hline \multirow{3}{*}{ MAz } & Sitting $>$ Treadmill & $0.000 *$ & \multirow{3}{*}{ High light $>$ Low light } & \multirow{3}{*}{$0.050 *$} \\
\hline & Sitting $>$ Walking & $0.000 *$ & & \\
\hline & Treadmill $=$ Walking & 1.000 & & \\
\hline
\end{tabular}

${ }^{a}$ Bonferroni confidence interval adjustment is used to adjust $\mathrm{p}$-values.

An asterisk (“*”) denotes a statistically significant difference at level 0.05. 


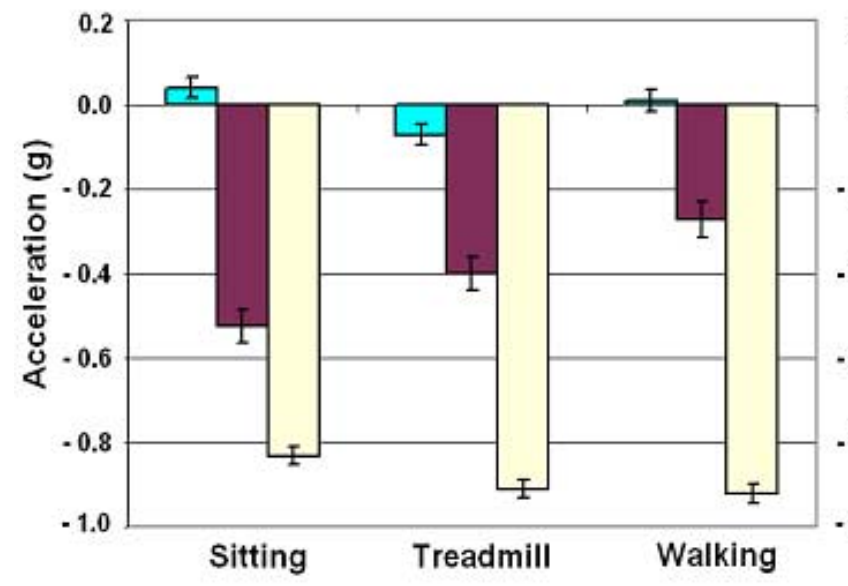

(a)

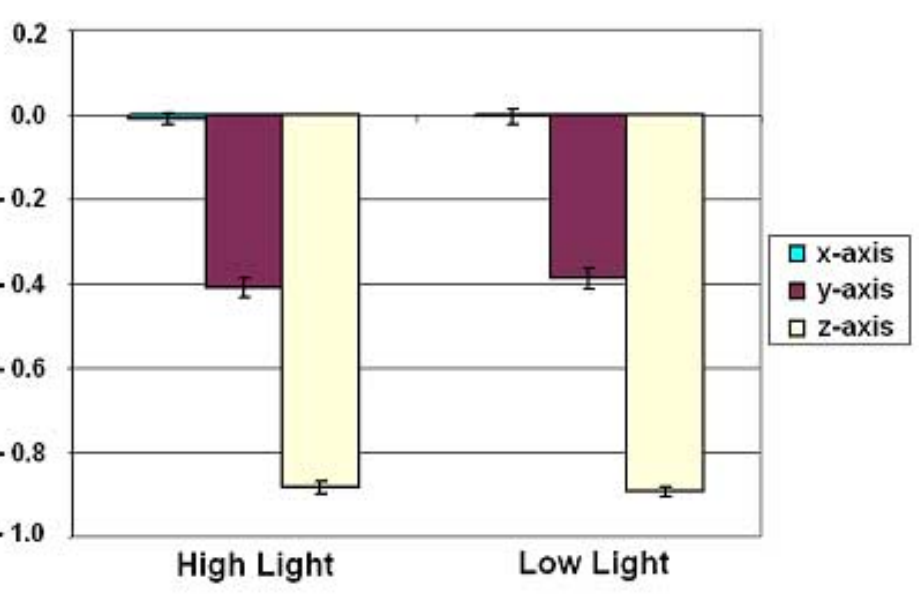

(b)

Figure 1. Estimated marginal means for means of acceleration for (a) mobility and (b) lighting conditions

\section{RESULTS}

The mean of acceleration was used because it is one of the most accessible measures of time series data [9]. Mean acceleration is composed of three axial components, the means of $\mathrm{x}$-axis (MAx), y-axis (MAy), and z-axis (MAz) movements. A multivariate two-way (3 mobility levels $\times 2$ light levels) repeated measures ANOVA with lighting as the within-subject factor was used. As shown in Table 2, the main effects of mobility and lighting were significant, and the interaction effect of lighting and mobility was also significant.

Based on the results of the multivariate ANOVA test, relationships between underlying measures and significant factors were investigated using pair-wise comparisons as shown in Table 3. The results indicate that, except for three pairs, sitting vs. walking in MAx, treadmill vs. walking in MAz and high light vs. low light in MAx, all pairs were significantly different.

Figure 1 shows the systematic trend more clearly. As the mobility condition became more complex, (from sitting to treadmill to path walking), MAy significantly increased. In contrast, MAz significantly decreased as the mobility condition was changed from sitting to treadmill. MAx's of the three mobility conditions are near zero and thus negligible compared to MAy and MAz. Additionally, changes to the lighting condition caused statistically significant differences. As the lighting changed from high light to low light, MAy significantly increased, while MAz significantly decreased.

\section{DISCUSSION AND CONCLUSIONS}

The results of the analyses show that the mobility and lighting factors systematically and significantly affected the output of the accelerometer. This may be explained by the fact that the mean of acceleration is determined by the orientation of the PDA, which is (in part) influenced by gravity. As shown in Figure 2, as the angle at which the PDA was held changed, gravity affected MAx, MAy and MAz differently. As users did not dramatically tilt the PDA toward the left or right during use, MAx was near zero with the primary affects on MAy and MAz.

This is consistent with observations made during experimentation. The participants in the walking condition tended to look down in order to see the path on the floor using their peripheral vision, so the PDA was tilted in the downward direction (Figure 2a). In the sitting condition, participants tended to orient it vertically instead (Figure 2c). In the treadmill condition, the orientation of the PDA was somewhere between (near $45^{\circ}$, Figure $2 \mathrm{~b}$ ). While the difference between conditions was smaller, the lighting factor also demonstrated similar trends. As the lighting was changed from high light to low light, MAy increased, but MAz decreased. This trend confirms that low lighting compelled participants to re-orient the PDA in the horizontal plane, in an attempt to gain as much exposure to light on the screen as possible, while also avoiding glare. Thus, the movement of the PDA under different contextual conditions, and its associated gravity effect, is revealed by the mean of acceleration measures.

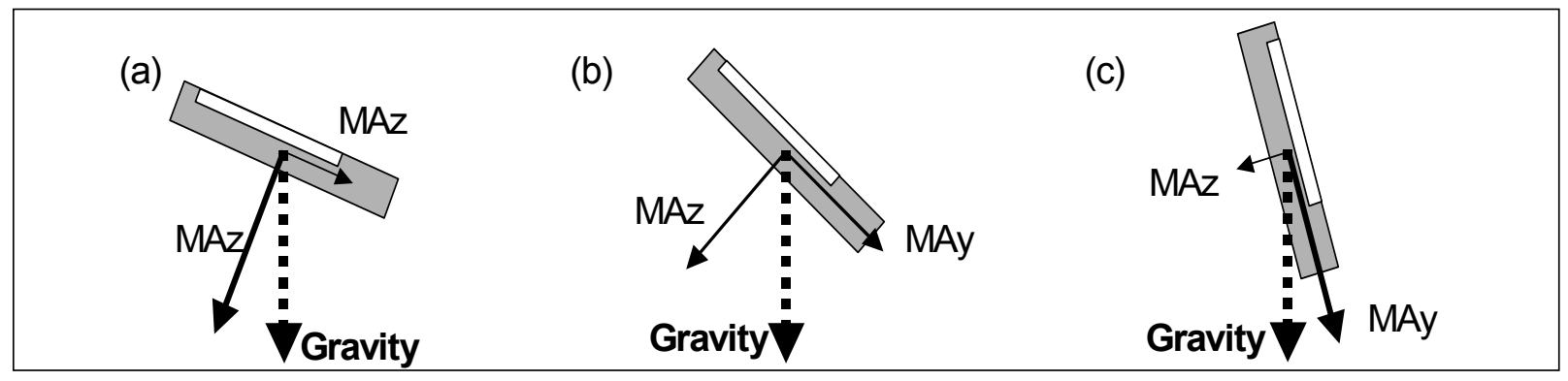

Figure 2. Side views of PDA in different angles 
The preliminary nature of this study introduced a few limitations, which should be acknowledged. First, the experiment was conducted in a laboratory setting and the tasks that participants performed were restricted to reading comprehension. Secondly, additional research will be needed to ascertain the implications of certain types of device movement in three-dimensions on realistic task scenarios, so that we can better understand user behaviors and design more effective interaction solutions. Finally, more advanced statistical analyses and/or additional sensors (e.g., microphone and ambient light sensor) coupled with a single accelerometer were not investigated, which remain as opportunities for future research.

The results of this experiment are encouraging because they show that mean acceleration for three axes from a single accelerometer can describe differences in movement related to actual user interaction with the device in context. Although the findings are limited to analyses of means, additional aspects of task context and movement could be revealed through ongoing examination of the experimental data with other measures and advanced statistical analyses. Using additional sensors also might enhance the robustness of collected contextual information.

\section{ACKNOWLEDGMENTS}

This material is based upon work supported by the National Science Foundation (NSF) under Grant No. IIS-0121570. The invaluable contributions of Leon Barnard, Paula Edwards, Thitima Kongnakorn, V. Kathlene Leonard, and Kevin Moloney are gratefully acknowledged.

\section{REFERENCES}

[1] Bao, L. Physical Activity Recognition from Acceleration Data under Semi-naturalistic Conditions. Massachusetts Institute of Technology, Cambridge, MA, M.Eng. Thesis Thesis, 2003.

[2] Bao, L. and Intille, S. S. Activity recognition from userannotated acceleration data in Proceedings of Pervasive 2004: the Second International Conference on Pervasive Computing (2004).
[3] Barnard, L., Yi, J. S., Jacko, J. A., and Sears, A. An Empirical Comparison of Use-in-Motion Evaluation Scenarios for Mobile Computing Devices. International Journal of Human-Computer Studies, 62, 4 (2005), 487520 .

[4] Barnard, L., Yi, J. S., Jacko, J. A., and Sears, A. A new perspective on mobile device evaluation methods. Personal and Ubiquitous Computing (in press).

[5] Cakmakci, O., Coutaz, J., Laerhoven, K. V., and Gellersen, H.-W. Context Awareness in Systems with Limited Resources in Proceedings of the third workshop on Artificial Intelligence in Mobile Systems (AIMS), ECAI 2002 (Lyon, France, 2002), 21-29.

[6] Chen, G. and Kotz, D. A Survey of Context-Aware Mobile Computing Research. Dept. of Computer Science, Dartmouth College TR2000-381, November 2000.

[7] Clayton, M. M. and Schamhardt, H. C., "Measurement Techniques for Gait Analysis", in Equine Locomotion, Back, W. and Clayton, H. M., Eds., 2000, pp. 400.

[8] Edmison, J., Jones, M., Lockhart, T., and Martin, T. An ETextile System for Motion Analysis in Proceedings of the International Workshop on a New Generation of Wearable Systems for eHealth, NGWSH (Lucca, Italy, Dec, 2003).

[9] Griffin, M. J. Handbook of human vibration. Academic Press, 1990.

[10] Kern, K., Schiele, B., and Schmidt, A. Multi-Sensor Activity Context Detection for Wearable Computing in Proceedings of European Symposium on Ambient Intelligence (EUSAI) (Eindhoven, The Netherlands, November, 2003).

[11] Kim, Y. H., Yi, J. B., and Rhee, K. H. Acceleration Patterns of the Body Center in Level Walking: Normal and Hemiplegic Patients. in Proceedings of World Congress on Medical Physics and Biomedical Engineering (Sydney, Australia, Aug 24-29, 2003), Paper No. 606. 